Le « printemps érable » au Québec : « Grève » ou « boycott »? Les enjeux stratégiques d'un conflit de nomination

The "Printemps Érable" in Quebec: "Grève" or "Boycott"? The Strategic Stakes of a Conflict of Naming

\title{
Gilles Gauthier
}

\section{OpenEdition Journals}

\section{Édition électronique}

URL : http://journals.openedition.org/aad/2248

DOI : 10.4000/aad. 2248

ISSN : 1565-8961

\section{Éditeur}

Université de Tel-Aviv

\section{Référence électronique}

Gilles Gauthier, « Le « printemps érable » au Québec : « Grève » ou « boycott » ? Les enjeux

stratégiques d'un conflit de nomination », Argumentation et Analyse du Discours [En ligne], 17 | 2016, mis en ligne le 15 octobre 2016, consulté le 23 septembre 2019. URL : http://journals.openedition.org/ aad/2248; DOl : 10.4000/aad.2248

Ce document a été généré automatiquement le 23 septembre 2019.

\section{cc) $(1) \odot$}

Argumentation \& analyse du discours est mis à disposition selon les termes de la licence Creative Commons Attribution - Pas d'Utilisation Commerciale - Pas de Modification 4.0 International. 


\title{
Le « printemps érable » au Québec: " Grève » ou « boycott »? Les enjeux stratégiques d'un conflit de nomination
}

\author{
The "Printemps Érable" in Quebec: "Grève" or "Boycott"? The Strategic Stakes of \\ a Conflict of Naming
}

Gilles Gauthier

Les débats politiques se résument trop souvent à des « dialogues de sourds » pour des pensées muettes.

Clément Frédéric Deville

\section{Introduction}

1 En 2012, survient au Québec ce qu'on a appelé «le printemps érable ». À son origine une cessation de cours décrétée par les associations étudiantes d'un certain nombre d'établissements universitaires et collégiaux en opposition à une augmentation des droits de scolarité, le mouvement se transforme rapidement en une contestation du gouvernement en place depuis une dizaine d'années et en une dénonciation généralisée du système capitaliste néo-libéral. L'une des questions principales posées durant la crise a concerné la désignation qu'il convenait de donner à l'arrêt des cours qui en a été le déclencheur. Les associations étudiantes et tous ceux, individus et organisations, qui les appuyaient, l'ont appelé "grève». Le gouvernement et les autres opposants à l'action étudiante l'ont plutôt baptisé « boycott ».

2 Je me propose ici d'examiner l'impact de ce conflit d'appellations dans le débat public du printemps érable. Le corpus analysé est constitué des textes publiés par les quatre principaux quotidiens francophones québécois - La Presse, Le Devoir, Le Journal de 
Montréal (Québec) et Le Soleil -, autant par les éditorialistes et chroniqueurs que par des intervenants extérieurs de février à septembre 2012. Leur nombre total s'élève à un peu plus de 800 ; toutes les occurrences des termes "grève » et «boycott» y ont été repérées. Leur examen mène à l'analyse ici proposée exemplifiée par des interventions parmi les plus représentatives. Je procéderai à une analyse à deux niveaux. Je ferai d'abord état de la portée stratégique de l'opposition entre les termes "grève " et «boycott » pour désigner l'arrêt de cours étudiant. J'élargirai après coup la perspective en analysant son effet sur la configuration initiale du débat sur la hausse des droits de scolarité. J'aborderai cette deuxième problématique à partir d'un cadre conceptuel articulé autour de l'idée d'amplitude du débat public.

3 L'examen de ce double effet de l'opposition d'appellations sera mené en regard de sa caractérisation préalable comme conflit sémantique. Je ferai voir, à ce propos, comment les dénominations "grève" et "boycott" sont parties prenantes d'un affrontement relatif à la représentation de la réalité. Comme les protagonistes en réfèrent eux-mêmes au dictionnaire et donnent ainsi à cette lutte pour la définition de la situation l'allure d'une dispute terminologique, une clarification lexicologique préalable permet de mieux l'appréhender.

\section{L'arrière-plan lexicologique}

4 Le dictionnaire reconnaît deux sens à chacun des termes "grève " et "boycott ». Le Petit Robert fournit de "grève » une première définition localisée et technique en la décrivant comme une

cessation volontaire et collective du travail, décidée par les salariés, un groupe professionnel dans un but revendicatif (augmentation de salaire, amélioration des conditions de travail, protestation contre les licenciements, etc.) et entrainant la suppression de la rémunération pendant cette période (1187).

Dans ce premier sens, est aujourd'hui accolée à « grève » une connotation légale que le dictionnaire marque en introduisant les notions de "Droit de grève » et "Grève illicite " (ibid.). La grève entendue dans ce sens restreint est donc une action réalisée dans un cadre juridique (pouvant varier d'un pays à l'autre et d'un secteur d'activité à un autre) relatif, entre autres choses, au vote de l'unité d'accréditation concernée et aux modalités de l'exercice du droit de grève.

6 L'autre sens donné à "grève » est beaucoup plus large ; c'est celui d'un "arrêt volontaire et collectif d'une activité par revendication ou protestation»(ibid.). Le dictionnaire illustre cette définition par les exemples «Grève des étudiants. Détenus qui se mettent en grève dans les prisons » et par l'expression « Grève de la faim » (ibid.). Ce second sens plus étendu de "grève » ne comporte pas la connotation légale du premier.

7 L'origine historique de " grève » rattache davantage le terme à son sens technique qu'à son sens large. Le mot tire son nom de la place de Grève à Paris, un point d'accostage des bateaux, elle-même ainsi baptisée parce qu'elle était bordée d'une plage de sable, où les hommes sans emploi cherchaient à se faire engager. "Faire grève " a ainsi d'abord signifié se tenir sur la place de Grève dans l'attente d'être embauché avant de désigner une cessation de travail quand des ouvriers mécontents se sont mis à s'y réunir pour protester contre leurs conditions d'emploi. C'est avec le temps qu'un cadre légal sera donné à ce type de débrayages. 
Quant au terme «boycott», le dictionnaire le définit d'abord globalement comme un «Interdit ou blocus matériel ou moral prononcé contre un individu, un groupe, un pays et contre les biens qu'il met en circulation " (ibid., 293). En marge de ce sens précis l'assimilant à un embargo, est également reconnu à "boycott » un sens plus large ${ }^{1}$, celui d'un «Refus de participer (à qqch.) ». En donnant l'exemple «boycotter des élections", le dictionnaire associe ce sens plus large de "boycott» à celui d'« abstention » (ibid.) $)^{2}$.

Dans la mesure où un refus de participer à quelque chose ou une abstention peuvent être considérés comme des cas d'espèce d'une cessation d'activité, les sens élargis de "grève » et de "boycott » sont apparentés. Cette similitude est avérée par l'origine historique de «boycott». Le terme tire son nom du patronyme de Charles Cunningham Boycott, un riche propriétaire terrien irlandais contre lequel les fermiers ont organisé un blocus allant jusqu'au sacrifice d'une récolte pour protester contre son refus de baisser le loyer des terres ${ }^{3}$. Comme ce blocus avait pour condition ou allait de pair avec une suspension d'activité, le boycott des fermiers a aussi été une grève au sens large d'une cessation collective de tâche comme moyen de revendication. Puisque cette revendication avait pour objectif de meilleures conditions de travail, ce premier boycott, bien que dénué de tout aspect légal, peut également être considéré comme le précurseur de la grève au sens strict.

Il ressort clairement de la considération terminologique qui précède que la distinction entre « grève » et «boycott " n'est pas clairement tranchée mais comporte plutôt un certain flou. Leur accointance de sens peut, relativement à certaines situations, ouvrir la voie à un usage équivoque. C'est sur le fond de cette ambiguïté que prend place le conflit d'appellations de la cessation de cours des étudiants lors du printemps érable.

\section{Un conflit référentiel}

11 Cette levée de cours n'est pas la première dans l'histoire du Québec. Elle s'ajoute à une longue liste de conflits ayant presque tous pour objet la revendication de la gratuité scolaire ou du gel des droits de scolaritét. Leur nombre élevé s'explique en bonne partie par la force des associations étudiantes québécoises due à leur reconnaissance légale. Il existe, en effet, au Québec depuis 1983 une «Loi sur l'accréditation et le financement des associations d'élèves ou d'étudiants » calquée partiellement sur le modèle de l'organisation syndicale en vigueur. En vertu de ce cadre législatif et réglementaire, une association se voit accorder le monopole de la représentation des étudiants ainsi que le droit de nommer les membres étudiants aux différents organismes des établissements collégiaux et universitaires en plus de bénéficier du prélèvement à la source d'une cotisation obligatoire (à l'image de la formule Rand) ${ }^{5}$. La Loi est toutefois complètement muette sur les prérogatives d'une association étudiante de déclencher une suspension des cours ainsi que sur l'encadrement de la démocratie étudiante. Elle ne reconnaît donc pas, ni ne dénie explicitement, un droit de grève aux associations étudiantes.

12 Néanmoins, les cessations de cours d'avant le printemps érable ont toujours été appelées "grèves » tout naturellement et sans que cette désignation soit mise en question. Cette admission spontanée peut s'expliquer de deux façons. Soit on concevait les levées de cours au sens large dépourvu de connotation légale de "grève ", donc comme une cessation d'activité par revendication ou protestation sans l'assimiler à un arrêt de travail de salariés encadré juridiquement - en exemplifiant la mention faite 
dans le dictionnaire de l'expression «Grève des étudiants ». Soit on les désignait plus ou moins métaphoriquement comme une "grève " au sens strict légal en considérant le statut quasi syndical des associations étudiantes même si son octroi ne les autorisait pas en toutes lettres à faire grève. Dans les deux cas, les débrayages étudiants pouvaient être dits des "grèves " sans que soit posée la question d'un droit de grève des associations étudiantes et de son encadrement juridique. De fait, la question de la désignation de la cessation de cours par les étudiants ne s'est pas posée et ne présentait donc aucun enjeu.

La situation change lors du printemps érable. Pour la première fois, le pouvoir politique vers lequel est dirigée la revendication étudiante et ceux qui le soutiennent dénient à l'action étudiante l'appellation de "grève ». En lieu et place, il la dénomme «boycott ». Selon toute vraisemblance, ce changement de nomination est d'abord motivé par une raison circonstancielle : le mouvement de levée de cours n'est pas suivi par la majorité des étudiants québécois et n'est pas non plus appuyé par l'ensemble de la population. La cessation de cours de 2012 a mis un certain temps à se mettre en branle et, bien que son action ait été importante et spectaculaire dans le temps, jamais tout au long du conflit, le regroupement des "carrés rouges", comme se baptisent ses tenants, ne réussit à atteindre $50 \%$ des étudiants des collèges et universités. Par ailleurs, tous les sondages font voir que la population québécoise est opposée, dans sa plus grande part, à la revendication étudiante. Les arrêts de cours antérieurs étaient ou avaient l'apparence d'être menés par une majorité d'étudiants et appuyés par l'ensemble des Québécois. Il y a fort à parier que le gouvernement n'aurait pas vu d'intérêt à chercher à imposer l'appellation «boycott » contre l'appellation "grève » si cela avait été aussi le cas lors de la cessation de cours de 2012. En la nommant «boycott" plutôt que " grève ", il cherche à profiter de la vulnérabilité relative avec laquelle elle se présente. En posant ce conflit d'appellations, le gouvernement poursuit manifestement une visée stratégique en provoquant un enjeu en marge de la question première de la hausse des droits de scolarité. C'est un enjeu de définition.

Comme H. de Chanay (2001) le met particulièrement bien en évidence, la dénomination procède d'un « désir de nomenclature " poursuivi dans l'interaction discursive. Dans le cas d'un conflit d'appellations, cette intention prend la forme d'un affrontement quant à la façon de désigner une réalité : l'opposition entre " grève » et " boycott " porte sur la nature de la cessation de cours des étudiants. De ce point de vue, le désaccord entre les tenants de la "grève » et ceux du "boycott » est d'ordre référentiel. Tel que le conçoivent les opposants, le conflit d'appellations consiste en une dispute sémantique ${ }^{6}$ au sens très classique de la sémiotique de Morris (1938), c'est-à-dire relative à la relation entre les signes, ici des termes, et les choses qu'on veut les voir signifier. On pourrait dire aussi, en recourant à la distinction de Frege (1971), que l'opposition entre " grève » et «boycott » porte sur deux sens donnés à la même dénotation, c'est-à-dire sur deux modes de présentation du même objet ${ }^{7}$. Pour chacun de leurs utilisateurs, les termes fournissent une représentation adéquate de la réalité. C'est même très précisément ce qui fonde leur désaccord : la levée de cours est une grève pour les uns et est un boycott pour les autres. Formellement, au point de départ, le conflit entre " grève » et «boycott » est une lutte pour faire prévaloir une désignation de la réalité sur une autre.

15 Le gouvernement cherche à faire jouer cette opposition en sa faveur. Exprimé sommairement, le raisonnement disjonctif, apparenté à une application du principe du 
tiers exclu, qu'il fait valoir est le suivant : comme les étudiants ne sont pas des salariés, une levée de cours, même décrétée par une association étudiante légalement reconnue, n'est pas assimilable à une cessation de travail reconnue juridiquement; ce n'est donc pas une "grève » au sens restreint à connotation légale, mais un "boycott » au sens large d'une abstention. Voici un exemple type d'expression gouvernementale de ce plaidoyer :

Peut-on s'entendre sur une chose : c'est pas une grève. Ça jamais été une grève. Non, c'est pas une grève. C'est parce qu'il faut s'entendre, faut employer les bons mots. Nous ne sommes pas des employeurs des étudiants. Les étudiants ne sont pas nos employés. C'est un boycott. Alors, employons les bons mots, là. C'est un boycott (le Premier ministre Jean Charest, cité par Legault 2012).

Le gouvernement se garde bien de considérer l'autre sens de " grève ", son sens large non légal de cessation d'une activité par revendication ou protestation. Mais même s'il le faisait, il pourrait encore faire valoir la minceur de la distinction entre ce sens élargi de "grève » et celui de «boycott » pour justifier son emploi de ce dernier terme. En ne marquant pas la différence entre les deux sens de "grève » il fait jouer à son avantage une option de nomination présentée comme dichotomique entre "grève " au sens strict et «boycott ».

17 Face à l'initiative gouvernementale de re-nomination, les associations étudiantes et ceux qui les appuient sont sur la défensive. Pour parer l'appellation «boycott» qui vient rompre la coutume jusque-là établie de désigner les suspensions de cours étudiants comme des " grèves ", le fardeau de la preuve leur revient. Elles doivent faire la démonstration que la simplification sémantique gouvernementale ne vaut pas. À cette fin, ils ont tout à la fois à faire valoir que la levée de cours est bien une grève et à marquer que ce n'est pas à la façon d'un boycott. La première partie de l'opération est aisée à mener. Il suffit d'invoquer le second sens de "grève" donné dans le dictionnaire. C'est tout particulièrement le cas de la politologue et chroniqueuse Josée Legault (2012) qui, après avoir fait état du sens strict légal de "grève » du Petit Robert, poursuit, en pointant explicitement son sens large :

Mais comme la langue française est riche, plusieurs autres définitions et exemples suivent quant à ses usages variés. Ainsi, on peut aussi y lire ceci comme définition : Arrêt volontaire et collectif d'une activité, par revendication ou protestation. [...] On dit bien "grève de la faim » et non «boycott de la faim » [...] Une grève étudiante est une grève étudiante.

Legault, à la suite, justifie l'usage de "grève » pour désigner les levées de cours des étudiants par une pratique historique généralisée :

En fait, depuis que les étudiants font grève sur cette terre, on parle en termes de " grève étudiante ». Le Québec ne fait pas exception au reste de la planète. Ailleurs ou ici, que ce soit en 2012, en 1958, ou lors de chacune des grèves étudiantes qui ont jalonné son histoire, en effet, on parle de grève et non de boycott. On le fait au prestigieux Le Monde. On le fait à Amnesty International. On le fait dans les médias. On le fait aux États-Unis. De nombreuses personnalités comme Claude Béland, Jean Cournoyer, Alain Vadeboncoeur, Robert Burns et Luc Picard, le font. Des intellectuels renommés, tel Guy Rocher, le font. La ligue des droits et libertés le fait. La commission des droits de la personne et des droits de la jeunesse du Québec le fait. On le fait en anglais. On parle de "strike ", pas de «boycott ». On le fait, entre autres, dans The Gazette, The Globe and Mail, The Toronto Star, The Wall Street Journal, The Guardian. On le fait en espagnol, en italien, en russe et dans toutes les langues. Etc., etc., etc. ... Conclusion. Le Québec n'est pas en situation de boycott étudiant. En fait, il vit la grève étudiante la plus longue et la plus élargie de toute son histoire. 
Les associations étudiantes et leurs appuis ne s'opposent de la sorte qu'indirectement à la définition gouvernementale, dans la mesure où ils font abstraction du contraste que celle-ci instaure entre le "boycott » et la "grève » entendue dans son sens strict à connotation légale. Mais ils ne peuvent pas se contenter de définir la cessation de cours comme une "grève " au sens large. Ils courraient alors le risque, étant donné la parenté entre les deux termes, de cautionner l'appellation «boycott». Aussi, cherchent-ils à en marquer la distinction. Afin de casser l'ambiguïté sémantique entre " grève » et «boycott ", ils avancent que la grève est collective alors que le boycott est individuel. Legault présente cette opposition en proposant une interprétation de l'intention gouvernementale du recours à « boycott » :

Et pourquoi au gouvernement, on insiste tant pour dire «boycott» et non " grève »? Pourquoi tenter aussi de convaincre les médias de faire de même?

Primo : parce qu'une grève découle d'une action collective.

Secundo : parce qu'au contraire, un boycott découle d'une action individuelle. Un individu boycotte quelque chose (ibid).

Le philosophe Christian Nadeau (2015) pour qui la nomination «boycott» est une manifestation de mépris à l'égard des étudiants ${ }^{8}$, fait la même distinction :

La différence entre une grève et un boycott est significative à plusieurs égards. [...] Un boycott est une action individuelle, qui repose sur des préférences personnelles. $\mathrm{Au}$ contraire, la grève découle de revendications collectives, pensées et formulées par un groupe. [...] De plus, le boycott relève d'un choix moral privé. Ce choix peut avoir des visées politiques, mais l'obligation qui y est attachée dépend du seul individu qui, dans une certaine mesure, n'a de compte à rendre qu'à lui-même (81).

21 Rien dans les définitions de "grève » et de «boycott » ne les différencie suivant cette ligne de partage. D'une part, si une « grève » au sens restreint légal est bien une action collective, elle peut être tout autant individuelle que collective au sens élargi du terme, qui est celui par lequel Legault et Nadeau voudraient voir désigner la cessation de cours. Une grève de la faim, par exemple, est le plus souvent le fait d'un seul individu. D'autre part, le boycott ne relève pas, lui, intrinsèquement d'une initiative personnelle. On peut certes dire, comme Legault, qu' " un individu boycotte quelque chose ", mais un «boycott», tant au sens premier d'un blocus qu'au sens plus étendu d'un refus de participer à quelque chose ou d'une abstention, peut tout à fait être mené par un groupe d'individus relativement à une revendication collective. Ainsi, le boycott d'élections est souvent appelé par un parti politique ou une autre organisation et constitue une conduite concertée. L'origine du terme indique également qu'un «boycott» peut être collectif: c'est bien regroupés que les fermiers de Charles Cunningham Boycott ont organisé contre lui le premier boycott. Finalement, bien que possible, un boycott qui resterait seulement individuel n'aurait pas une grande signification ou portée sociale.

La levée étudiante de cours est bien sûr collective. Mais cela n'en fait pas une "grève » plutôt qu'un «boycott ». Le prétendre, comme le font Legault et Nadeau, c'est occulter l'extension individuelle possible de "grève " au sens élargi d'une cessation d'activité par revendication ou protestation et l'extension collective possible de «boycott » au sens second d'un refus de participer à quelque chose ou d'une abstention. Ce double aveuglement s'explique par le fait que, dans le contexte du printemps érable, contrairement à «boycott » qui relève d'une conceptualisation rudimentaire, "grève " s'avère d'un maniement lexical beaucoup plus subtil et complexe. Comme le fait voir la citation du Premier ministre Charest rapportée plus haut, dès que la cessation de cours 
peut être différenciée d'un arrêt de travail du fait que les étudiants ne sont pas des " employés » ni le gouvernement leur "employeur ", on peut lui dénier l'appellation "grève » au sens strict légal et proposer en lieu et place celle de «boycott ». Certains de ceux qui appuient la position gouvernementale poursuivent le raisonnement comparatif :

Les salariés qui font la grève retirent leur force de travail et, en contrepartie, peuvent être victimes d'un lock-out, sans compter qu'ils sont pendant ce temps privés de salaire. [...] Les étudiants sont les bénéficiaires d'un service public. Ils ont le droit de refuser ce service, mais cela n'en fait pas des grévistes, pas plus qu'un groupe de bénéficiaires des services de santé qui décideraient de boycotter leur hôpital (Gagnon 2015 : A15).

Comme noté plus haut, les choses ne sont pas aussi simples pour ceux qui veulent malgré tout considérer l'arrêt de cours comme une grève. Forcés de concéder qu'il n'est pas une suspension de travail, ils doivent non seulement faire valoir le sens étendu de "grève ", mais également marquer qu'il n'est pas assimilable à celui de "boycott ». C'est en introduisant la distinction erronée entre action collective et action individuelle qu'ils cherchent à le faire.

24 La manœuvre illustre bien la difficulté sémantique dans laquelle se trouvent les partisans de l'arrêt de cours. Elle se manifeste également dans le manque de définition, de description et même de discussion avec lequel ils abordent parfois le conflit d'appellations entre "grève» et «boycott». Par exemple, dans le Dictionnaire de la révolte étudiante publié après le printemps érable par des sympathisants de la cause étudiante, les entrées «boycott " et "grève " sont caractérisées essentiellement de façon ironique (Deslauriers 2012 : 27-28 et Farah 2012 : 89-90).

\section{Une portée stratégique}

Le fait que le conflit d'appellations entre " grève et «boycott " relève d'une opposition sémantique ne l'abstrait pas du débat public. Le recours à l'un ou l'autre terme, loin d'être innocent et neutre, est au contraire directement en rapport avec des choix politiques et idéologiques. Seulement, ce lien, pour reprendre la conceptualisation de Morris, est d'ordre pragmatique plutôt que sémantique : il a trait à la relation des termes à leurs utilisateurs, c'est-à-dire à l'usage auquel servent " grève » et « boycott ». Il y a une corrélation entre la façon dont l'arrêt de cours étudiant est défini et les positions prises sur l'augmentation des droits de scolarité. Ce sont ceux qui appuient la hausse qui désignent le débrayage étudiant comme un "boycott» et ceux qui s'opposent à la hausse qui le désignent comme une « grève ».

Cette concordance ne relève cependant pas d'une contrainte logique. Il n'y a pas incompatibilité formelle entre chacune des appellations et la position contraire à celle à laquelle elle est liée. Il aurait été parfaitement possible qu'un opposant à la hausse considère la cessation de cours comme un «boycott » et, à l'opposé, qu'un partisan de la hausse la définisse comme une " grève ».

L'engagement pragmatique de la nomination n'est donc pas linéaire. Même si, de fait, ceux qui désignent la levée de cours des étudiants comme une "grève " sont contre la hausse et ceux qui la désignent comme un «boycott » sont en sa faveur, la position des uns et des autres n'est pas fondée sur leur nomination respective. En toute logique, l'opposition à l'augmentation des droits de scolarité ne repose pas sur le fait que la 
cessation de cours des étudiants soit une « grève " ni l'appui à cette hausse qu'elle soit un « boycott ». La nomination n'est pas, en tout cas pas toujours, partie prenante dans l'argumentation centrale d'un débat. Son impact se situe ailleurs.

Le conflit d'appellations entre "boycott » et "grève » a trait à la légitimité de la revendication étudiante. Comme l'ont souligné un grand nombre d'observateurs et de critiques (parmi d'autres, Rocher 2012 et Dufour 2015), l'appellation gouvernementale «boycott » a pour objectif de délégitimer l'opposition des étudiants à la hausse de droits de scolarité.

Ceux pour qui la distinction entre la grève et le boycott en est une entre une action collective et une action individuelle avancent que cette tentative de délégitimation porte plus précisément sur la nature démocratique de la cessation de cours. C'est d'ailleurs très précisément parce qu'ils attribuent au gouvernement le dessein de dénier ce caractère démocratique qu'ils introduisent la distinction. Josée Legault affirme ainsi que parce qu'elle relève d'une action collective, la grève « découle donc d'une décision prise démocratiquement par des citoyens regroupés ou associés » et, conséquemment, que " parler de boycott envoie un message bien précis à l'électorat: ce mouvement étudiant n'est pas vraiment démocratique... » (op. cit.). Christian Nadeau reprend l'argument en affirmant que parce qu'il se distingue de la "grève » en ce qu'il ne dépend pas de revendications collectives mais plutôt de préférences personnelles, par le choix du terme "boycott ", "c'est également la dimension démocratique de la grève qui est évacuée, car le choix d'entrer en grève serait un geste privé, décidé unilatéralement, et non le résultat d'une discussion démocratique publique suivie d'un vote » (op. cit.).

Dans la mesure où l'opposition entre action collective et action individuelle ne vaut pas pour marquer la distinction entre la grève et le boycott, ce ne peut être, d'un point de vue logique, pour la discréditer au plan démocratique, contrairement à ce qu'en disent Legault et Nadeau, que le gouvernement choisit d'appeler la cessation de cours des étudiants un "boycott " plutôt qu'une "grève ». Si, en son sens élargi d'un refus de participer ou d'une abstention (comme en son sens premier d'un blocus, d'ailleurs) un «boycott " peut relever d'une revendication collective, on ne voit pas en quoi il serait moins démocratique qu'une grève et, par suite, comment, en toute logique, préférer le terme « boycott » à celui de " grève » reviendrait à priver l'arrêt de cours des étudiants d'un caractère démocratique. Comme une grève, un boycott peut tout à fait découler d'une discussion et d'un vote au sein d'une assemblée et ne pas dépendre d'une décision privée. C'est le cas des boycotts d'élections décrétés par un parti politique ou une autre organisation. Rien, donc, ne distingue formellement du point de vue démocratique le boycott et la grève. Qu'on qualifie la cessation des cours du printemps érable de «boycott » ou de " grève », que ce soit aux sens restreints ou aux sens plus larges des deux termes, n'affecte en rien la nature démocratique dont elle est pourvue ou dépourvue. Elle est ou elle n'est pas démocratique pour des raisons ou des considérations qui restent étrangères à l'appellation qu'on choisit pour la dénoter. Lier les termes de «boycott » et de " grève » dans un sens ou l'autre à la démocratie relève d'une erreur catégorielle.

31 Si Legault et Nadeau imputent au gouvernement l'intention de nier la nature démocratique de la levée de cours des étudiante, c'est parce qu'ils se trouvent dans une situation de double contrainte définitionnelle : forcés d'admettre que la suspension de cours n'est pas une grève au sens strict d'un arrêt de travail par des employés, ils 
veulent lui préserver l'appellation «grève » au sens large sans connotation légale ... tout en cherchant à lui prêter une certaine portée légale. On trouve un indice de cette tension dans un dernier élément avancé par Nadeau au sujet du boycott : «le boycott relève d'un choix moral privé. Ce choix peut avoir des visées politiques, mais l'obligation qui y est attachée dépend du seul individu qui, dans une certaine mesure, n'a de compte à rendre qu'à lui-même » (ibid.). Comme il s'agit là d'un trait par lequel le boycott se distingue de la grève, on doit comprendre que l'obligation rattachée à celleci ne dépend pas du seul individu et que ce dernier est redevable au groupe dans lequel sont "pensées et formulées [les revendications collectives desquelles] la grève découle " (ibid.). Or, la grève comportant cette obligation ne peut être que la "grève " au sens plus strict d'une cessation de travail par des employés encadrée légalement. Ce n'est en effet que dans ce type de grève que l'individu ne peut pas de lui-même mettre fin à l'arrêt de travail, que la reprise du travail ne peut, comme sa suspension, être décidée que collectivement. Dans le cas d'une "grève" au sens large d'un arrêt volontaire d'une activité par revendication ou protestation dénué de connotation légale, comme une grève de la faim, une grève de détenus dans les prisons et une grève d'étudiants (pour reprendre de nouveau les exemples donnés pas le dictionnaire), cette obligation ne fait l'objet d'aucune reconnaissance ni même spécification légale. Afin de préserver ce terme de "grève " au sens élargi pour désigner la cessation de cours, Nadeau finit ainsi par lui attribuer une caractéristique de la "grève » au sens strict légal dont il cherche portant à la distinguer.

Il est tout à fait clair que le gouvernement et les autres opposants à la cessation de cours des étudiants ont mis en cause son caractère démocratique. Mais, contrairement à ce qu'en disent Legault et Nadeau, ils ne l'ont pas fait en l'appelant un «boycott ». Ils ne pouvaient logiquement le faire en regard du sens du terme qui, encore une fois, n'implique pas qu'un boycott soit moins démocratique qu'une grève. C'est en incriminant la façon dont ont été tenues les assemblées générales des associations étudiantes et tout particulièrement les votes qui y ont été pris que le gouvernement a contesté la représentativité de la levée de cours, en prétendant qu'elle a toujours résulté de la volonté d'une minorité d'étudiants.

En la dénommant «boycott», le gouvernement et ceux qui l'appuient poursuivent une autre visée stratégique que celle de la prétendre non démocratique. L'enjeu au cœur du conflit d'appellations entre "boycott " et "grève » a trait à la judiciarisation du débrayage étudiant. Le recours à «boycott» sert à amputer la cessation de cours de toute composante légale et ainsi à la délégitimer en en récusant la nature juridique. Jusqu'au printemps érable, comme noté précédemment, les cessations de cours des étudiants étaient appelées " grève " sans qu'il soit indiqué dans lequel de ses deux sens il convenait d'entendre l'usage du terme. Cette équivoque permettait de laisser en suspens la question du caractère légal et de la portée juridique des levées de cours. En la dénommant "boycott», le gouvernement cherche à dénier à celle de 2012 toute dimension légale. Considérée comme un "refus de participer » ou une "abstention ", elle est dépouillée de statut juridique et de la sorte réduite à une réclamation désordonnée, sinon carrément anarchique. C'est un objectif de délégitimation légale et non pas démocratique que poursuivit le gouvernement en recourant à l'appellation "boycott ». En définissant la cessation de cours comme une "grève ", on reconnaît, même confusément, qu'elle est ou qu'elle peut être située dans une zone d'application du droit ; en la qualifiant plutôt de "boycott », on l'exclut de ce champ d'application. Cette disqualification a pour double effet de priver la revendication étudiante de tout 
recours juridique, et au gouvernement de revendiquer pour lui seul la légalité. Elle justifie également son refus de négocier avec les associations étudiantes.

Le gouvernement aurait pu poursuivre la même visée stratégique en faisant valoir qu'une suspension de cours n'est pas assimilable à une "grève » au sens premier du terme, mais seulement en son sens second dénué de connotation légale de l'arrêt d'une activité par revendication ou protestation. Mais, outre que l'emploi de «boycott » est plus simple et plus efficace, il se serait alors lui-même placé dans une situation gênante semblable à celle à laquelle sont confrontées les associations étudiantes et ceux qui les appuient. Il aurait eu à faire la démonstration de la distinction entre la "grève » au sens large et la " grève " au sens strict. Le gouvernement élude la difficulté en tablant sur l'opposition entre "grève » et «boycott ». Les partisans de la cessation de cours ne peuvent en faire autant: ils sont acculés à soutenir que la levée de cours est une " grève " au sens élargi en le distinguant de son sens strict, quitte à tenter parfois de faire marche arrière, comme Christian Nadeau quand il assigne à la "grève » au sens large une obligation propre à la "grève » au sens restreint dont il veut néanmoins la différencier. Ils laissent ainsi le gouvernement et ceux qui le soutiennent occuper tout le terrain juridique. Si la levée de cours n'est pas une "grève ", elle n'implique pas l'exercice d'un droit de grève et s'il n'y a pas droit de grève, ceux qui s'y opposent peuvent faire valoir leur droit à suivre leurs cours. À la cessation de cours se sont ainsi opposés des étudiants qui ont revendiqué le droit de recevoir les enseignements auxquels ils étaient inscrits et pour lesquels ils avaient acquitté les droits. A cette fin, une vingtaine d'entre eux ont obtenu des tribunaux des injonctions qui, à défaut d'être respectées, ont judiciarisé le printemps érable". S'est aussi constitué un "mouvement des étudiants socialement responsables ", dit des "carrés verts ", qui ont manifesté leur appui à l'augmentation des droits de scolarité.

\section{Un effet de majoration d'amplitude}

En plus de poser la question de la légitimité de l'arrêt de cours étudiant, le conflit d'appellations entre « boycott » et " grève » modifie de façon sensible le cadre même de l'affrontement entre adversaires et défenseurs de la hausse des droits de scolarité. Il est possible d'en rendre compte en mobilisant la notion d'« amplitude » du débat public.

36 La saisie la plus immédiate qu'on a habituellement d'un débat le montre comme un ensemble formellement organisé. Cette représentation structurelle n'est pas totalement inadéquate : un débat est bel et bien agencé suivant des cadres repérables. Il porte sur une question relativement précise, à propos de laquelle sont adoptées des positions globalement bien identifiées, suivant des lignes d'opposition qu'il est également possible de déterminer avec suffisamment de précision. Un débat public n'est toutefois pas qu'un ensemble statique, mais est au contraire animé d'une certaine dynamique. S'il est possible d'en fournir une analyse structurelle en établissant ses tenants et aboutissants, les désaccords sur lesquels il porte ainsi que les interactions sociales dont il procède et qu'il met en marche, le débat ne se réduit pas à cet agencement ordonné. Un débat bouge ; il est mû par certains facteurs qui viennent bousculer son état d'équilibre provisoire et réorganiser sa structuration.

37 La dynamique du débat est faite de deux composantes : sa mobilité et son amplitude. La mobilité du débat peut être elle-même de deux types. Elle peut consister en un déplacement de son objet occasionné par des modifications des perspectives 
doctrinales, idéologiques ou politiques, à partir desquelles il est abordé ou encore du contexte dans lequel il se présente. Chateauraynaud (2011a) montre bien, par exemple, comment le débat sur l'énergie nucléaire en Europe a été remanié par des retournements dans la considération de l'argument climatique. Ainsi, comme Chateauraynaud encore (2011b) le décrit, un débat peut avoir une trajectoire inscrite dans une chronologie.

La mobilité du débat peut également consister en un déplacement d'enjeux dans le temps. Au Québec, par exemple, le débat sur le mariage gai a dérivé au fil de son cheminement et de sa résolution vers celui de l'adoption d'enfants par les couples homosexuels (Gauthier 2012).

Le deuxième constituant de la dynamique du débat est non pas diachronique, mais synchronique. C'est son amplitude. Même vu en une coupe temporelle, un débat n'a pas une configuration fixe. Ses paramètres, loin d'être rigides, font l'objet d'une certaine oscillation. L'amplitude du débat, c'est en quelque sorte l'élasticité que lui fait acquérir la diversité des interventions dont il fait l'objet. Pour exprimer les choses d'une manière plus conceptuelle, l'amplitude du débat, c'est la variation dont sa teneur est affectée par suite des accentuations, altérations, déviations et autres glissements ou écarts que lui impriment les prises de positions différentes qu'il suscite (Gauthier 2014). L'amplitude d'un débat fait que son déploiement, même en un certain état et à certain moment, est à géométrie variable.

Il peut arriver que l'amplitude d'un débat soit minimale et il est même théoriquement possible qu'elle soit nulle. Il faut, pour que ce soit le cas, que les points de vue opposés qui y sont défendus ne fluctuent pas de manière significative ou restent même parfaitement immobiles. Cela ne survient que dans des situations exceptionnelles, sinon idéalisées de discours. La plupart du temps, les débats publics connaissent des mouvements d'amplitude qui en élargissent ou en rétrécissent le champ et qui ainsi les dilatent ou les contractent.

41 Plusieurs facteurs distincts peuvent venir modifier l'amplitude d'un débat. L'un des plus fréquents est la dissymétrie entre les raisons et autres considérations invoquées à l'appui des positions prises dans un débat (Gauthier 2016a). Cette dissymétrie survient quand ces raisons et considérations sont en discordance les unes par rapport aux autres. Le débat, alors, perd de son unité en se dispersant sur des terrains différents. La dissymétrie peut avoir diverses causes. La plus fréquente est une divergence d'interprétation relative à un élément mis en cause dans un débat ${ }^{10}$. Le conflit de nomination entre "boycott" et "grève " relève de cette catégorie générale. En définissant par l'un ou l'autre termes la cessation de cours des étudiants, les intervenants au débat y introduisent une distorsion qui brise son équilibre initial en le projetant sur un nouveau point de controverse.

La majoration d'amplitude générée par le conflit de nomination entre "grève " et «boycott » dépasse cependant ce simple effet d'élargissement. Jusqu'à un certain point, l'affrontement sur la façon dont il convient de désigner l'arrêt de cours disloque le débat sur la hausse des droits de scolarité. En s'insinuant dans le débat sur la hausse des droits de scolarité, le conflit de nomination le vampirise. Au point de départ, le désaccord sur la nature de la cessation de cours vient simplement se greffer à l'affrontement initial portant sur cette hausse et agrandir le champ de la discussion. L'amplitude du débat s'en trouve tout naturellement accrue : ce sont, dès lors, deux questions qui font l'objet du débat et mettent aux prises les mêmes intervenants. Mais 
le conflit d'appellations entre "grève » et "boycott " ne reste pas une simple annexe du débat d'origine. Il exerce sur lui un effet de décentrement. Pendant le temps passé à débattre de la question de la définition de la cessation de cours, on ne discute plus du bien-fondé de la hausse des droits de scolarité. En s'y plaquant, le conflit d'appellations tend ainsi à reléguer dans l'ombre le débat sur la hausse des droits.

Comme à chaque fois qu'un débat connaît une majoration d'amplitude produite par dissymétrie (Gauthier 2016a), ce décalage et la marginalisation qu'elle entraine ont également pour effet d'obscurcir la discussion. La compréhension des véritables enjeux d'une augmentation des droits de scolarité, sociaux et économiques, se perd dans l'affrontement sur la question de savoir si la cessation de cours des étudiants est une " grève » ou un « boycott ». Cet obscurcissement favorise une polarisation des positions qui rigidifie le débat. Il devient un dialogue de sourds où chacun non seulement ne cherche plus à bien comprendre le point de vue opposé, mais n'en tient même plus compte dans la détermination du sien. La dimension conflictuelle du débat écrase sa dimension dialogique.

\section{Conclusion}

Le débat sur la hausse des droits de scolarité a officiellement pris fin en septembre 2012 quand un nouveau gouvernement (élu au début du mois) annule l'augmentation des droits de scolarité. En février 2013, se tient un « Sommet sur l'enseignement supérieur au Québec » au terme duquel le même gouvernement annonce une indexation des droits à l'augmentation du revenu disponible des familles.

Par ailleurs, les suites du printemps érable ont vu surgir un nouveau débat sur la reconnaissance d'un droit de grève aux associations étudiantes. Dans son rapport (Bissonnette et Porter 2013), le Chantier sur une loi-cadre des universités, lancée lors du Sommet, propose que soient mieux définies les règles présidant aux arrêts de cours étudiants ainsi que les conditions de validation des résolutions votées par les associations étudiantes. De manière plus explicite, le rapport de la Commission spéciale d'examen des événements du printemps 2012 mise sur pied par le gouvernement (Ménard, Grenier et Carbonneau 2014) recommande que soit légalement reconnu aux associations étudiantes un droit de grève dont l'exercice serait balisé par des règles strictes $^{11}$. Cette recommandation fait l'objet d'une controverse prenant diverses formes au sein des associations étudiantes, des différents partis politiques et dans la presse quand a lieu, en 2015, une tentative de relancer un nouveau printemps érable ${ }^{12}$.

Ce débat sur l'octroi d'un droit de grève étudiant (qui est toujours en suspens) est une résultante directe du conflit de nomination entre "grève » et «boycott » : c'est parce que l'opposition entre les deux appellations a fondamentalement trait à la dimension légale des levées de cours que l'affrontement auquel elle donne lieu finit par déboucher sur la question de l'à-propos de reconnaître aux associations étudiantes un droit de grève analogue à celui des associations syndicales. Il s'agit là d'une manifestation de mobilité du débat public. L'enjeu de la reconnaissance d'un droit de grève s'est substitué, dans le temps, à celui de la façon appropriée de définir l'arrêt de cours étudiant et aussi, transitivement, à celui de la hausse des droits de scolarité. Le débat du printemps érable québécois de 2012 a ainsi été le lieu d'une dynamique particulièrement puissante dont l'origine et le moteur ont été le conflit de nomination entre « grève » et « boycott ». 


\section{BIBLIOGRAPHIE}

Anscombre, Jean-Claude. 2001. « Dénomination. Sens et référence dans une théorie des stéréotypes nominaux ", Cahiers de Praxématique 36, http://praxematique.revues.org/304, consulté le 3 octobre 2015

Bissonnette, Lise et John R. Porter. 2013. « L'Université québécoise : Préserver les fondements, engager des refondations. Rapport du Chantier sur une loi-cadre des universités » (Québec : Ministère de l'Enseignement supérieur, de la Recherche, de la Science et de la Technologie)

Chateauraynaud, Francis. 2011a. « Sociologie argumentative et dynamique des controverses : l'exemple de l'argument climatique dans la relance de l'énergie nucléaire en Europe », A contrario $16,131-150$

Chateauraynaud, Francis. 2011b. Argumenter dans un champ de force. Essai de balistique sociologique (Paris : Pétra)

Chanay, Hughes Constantin de. 2001. « La dénomination : perspective discursive et interactive, Cahiers de Praxématique 36, http://praxematique.revues.org/358, consulté le 3 octobre 2015

Deslauriers, Félix L. 2012. « Boycott », Isabel, Marie-Ève et Laurence-Aurélie Théroux-Marcotte (eds), Dictionnaire de la révolte étudiante (Montréal : Tête première), 27-28

Dufour, Pascale. 2015. «La rue contre les urnes? Mouvement étudiant et représentation politique ", Tremblay, Pierre-André, Michel Roche et Sabrina Tremblay (eds), Le printemps québécois. Le mouvement étudiant de 2012 (Québec : Presses de l'Université du Québec), 89-103 Farah, Alain. 2012. « Grève », Isabel, Marie-Ève et Laurence-Auérlie Théroux-Marcotte (eds), Dictionnaire de la révolte étudiante (Montréal : Tête première), 89-90

Frege, Gottlob. 1971 (1892). « Sens et dénotation », Écrits logiques et philosophiques (Paris : Le Seuil) Gagnon, Lysiane. 2015. « La fiction du 'droit de grève’ étudiant », La Presse, 14 avril

Garon, Claude. 2000. « Le mouvement étudiant québécois bénéficie d'une loi unique au monde », Cité Éducative 15 (2), 16-17

Gauthier, Gilles. « Le débat sur la charte québécoise de la laïcité : un brouillage produit par la diversité des conceptions du rapport entre espace civique et espace public », à paraître [1] dans Religiologiques

Gauthier, Gilles. « La mise en débat de l'événement par la blogosphère journalistique », à paraître [2]

Gauthier, Gilles. 2016a. « Dissymétrie et amplitude dans le débat contemporain », Argumentum 14 (1), 7-30

Gauthier, Gilles. 2016b. « La prétention à la vérité de l'argumentation morale dans le débat public. Un cas paradigmatique : la justification de la justice sociale », Thion Soriano-Molla, Dolores, Noémie François et Jean Albrespit (eds), Fabriques de vérité(s). Communication et imaginaires, vol.1 (Paris : L'Harmattan), 103-116

Gauthier, Gilles. 2015. «L'effet de l'argument de comparaison dans le débat public », Myriades 1, http://cchum.ilch.uminho.pt/myriades\#courant, consulté le 27 septembre2015

Gauthier, Gilles. 2014. « L'amplitude du débat public », Studies in Communication Sciences 14, 129-135 
Gauthier, Gilles. 2013. « L'argumentation morale dans le débat public. Une confrontation asymétrique », Ethica 18 (1), 119-145

Gauthier, Gilles. 2012. « La moralisation du débat public. Structuration des arguments moraux », Communication \& Langages $172,97-118$

Lacoursière, Benoit. 2012. «Grève ou Boycott », Profs contre la hausse, 11 avril, http:// profscontrelahausse.org/billets/greve-ou-boycott, consulté le 28 septembre 2015

Legault, Josée. 2012. « Ceci n'est pas une grève ", L’Actualité, 7 août, http://www.lactualite.com/ blogues/le-blogue-politique/ceci-nest-pas-une-greve, consulté le 28 septembre 2015

Ménard, Serge, Bernard Grenier et Claudette Carbonneau. 2014. «Rapport. Commission spéciale d'examen des événements du printemps 2012 » (Québec : Gouvernement du Québec)

Morris, Charles W. 1938. Foundations of the Theory of Signs (Chicago: University of Chicago Press)

Nadeau, Christian. 2015. « Autonomie, mépris et reconnaissance », Tremblay, Pierre-André, Michel Roche et Sabrina Tremblay (eds). Le printemps québécois. Le mouvement étudiant de 2012

(Québec : Presses de l'Université du Québec), 73-88

Trudel, Pierre. 2014. « La liberté d'association des étudiants », Le Journal de Montréal, 11 décembre

\section{NOTES}

1. À strictement parler, le Petit Robert considère qu'il s'agit d'une nuance de sens.

2. Il s'agit là, en fait, des définitions de "boycottage ", "boycott » étant considéré comme un anglicisme par le Robert. Bien que «boycottage » soit normativement plus correct, «boycott » fait l'objet d'un usage courant au Québec comme dans l'ensemble de la francophonie.

3. Wiktionnaire : https://fr.wiktionary.org/wiki/boycott

4. Pour un survol de ces conflits, voir l'historique établi par la CLASSE (la Coalition large de l'Association pour une Solidarité syndicale étudiante) : http://www.bloquonslahausse.com/ verslagreve/historique-des-greves-generales/

5. Comme le notent des observateurs, le mouvement étudiant québécois jouit ainsi d'une reconnaissance légale unique au monde (Dufour 2015 : 99 et Garon $2000: 16-17$ ).

6. Comme le note d'ailleurs de Chanay, qui en propose une analyse relative à la tension entre langue et discours, « la notion de dénomination nous place dans la perspective d'une sémantique référentielle » (ibid., 1).

7. Cette façon de présenter les choses laisse évidemment ouverte toutes les questions philosophiques et linguistiques relatives à cette fonction référentielle telles que les caractérise Anscombre, par exemple (2001).

8. Nadeau, s'inspirant d'Axel Honneth (La lutte pour le reconnaissance) et d'Emmanuel Renault (L'expérience de l'injustice. Reconnaissance et clinique de l'injustice), propose une analyse philosophique du printemps érable suivant laquelle la lutte étudiante en était une pour l'autonomie et la reconnaissance à laquelle le gouvernement, les médias et des directions d'établissements collégiaux et universitaires n'auraient réagi qu'avec mépris.

9. Quelques-uns de ces étudiants ont également par la suite contesté la validité constitutionnelle de la Loi sur l'accréditation et le financement des associations étudiantes au motif qu'elle violait les droits à la liberté d'association et à la liberté d'expression garanties par la Charte canadienne des droits et libertés. Le principal argument présenté à l'appui de leur cause était que la transposition du fonctionnement des syndicats au monde étudiant est inadéquate. Ils ont ainsi été amenés à contester frontalement le recours à l'appellation « grève ", entendue dans son sens 
premier d'une cessation du travail par des employés, pour désigner un débrayage étudiant (voir Trudel 2014).

10. Quelques-uns de ses autres types sont la dissemblance conceptuelle : la référence à un même concept, mais entendu dans des sens distincts (Gauthier 2016b) ; la confusion conceptuelle : des rapports différents entre deux concepts apparentés (Gauthier, à paraître 1) et l'attribution de sens extrinsèque : des interprétations concurrentes indues données à un événement (Gauthier, à paraître 2). D'autres causes fréquentes de dissymétrie sont le recours aux justifications morales et l'usage de la comparaison (Gauthier 2013 et 2015) qui, incidemment, a aussi, mais différemment, exercé un effet d'amplitude sur le débat sur les droits de scolarité.

11. Dans la mesure où cette proposition présuppose que les associations étudiantes n'ont pas déjà ce droit de grève, elle constitue un indice de l'efficacité de la stratégie gouvernementale lors du printemps érable : l'emploi de "boycott" a réussi à imposer l'idée que la levée de cours étudiante n'était pas une " grève » au sens premier du terme.

12. À cette occasion, la levée de cours a été baptisée "grève sociale " par ses organisateurs d'abord parce qu'elle ne portait pas sur une revendication bien précise comme l'augmentation des droits de scolarité. Cette appellation avait en outre l'avantage de contrer l'ambiguïté posée par l'opposition entre " grève » et « boycott » en attribuant d'emblée et explicitement à " grève » son sens second sans connotation légale d'un arrêt d'activité par protestation.

\section{RÉSUMÉS}

Lors du printemps érable québécois 2012, la cessation de cours décrétée par les associations étudiantes dans des établissements universitaires et collégiaux pour protester contre une hausse des droits de scolarité a été baptisée " grève » par les uns et «boycott » par les autres. Ce conflit de nomination, qui a fait fond sur un arrière-plan terminologique, a essentiellement consisté en un affrontement sémantique. Il fait l'objet d'une double analyse : de sa portée stratégique et de son effet sur la configuration du débat sur la hausse des droits de scolarité à l'aide d'un cadre conceptuel développé à partir de la notion centrale d'« amplitude » du débat public.

During the "printemps érable québécois" of 2012, the annulment of courses decreed by the student associations in universities and colleges to protest against an increase in tuition fees was baptized a "strike" by some and a "boycott" by others. This conflict of naming, which builds on a terminological background, consisted mainly in a semantic confrontation, can be analysed in two ways: in terms of its strategic impact and in terms of its effect on the configuration of the debate on rising tuition, based on a conceptual framework developed from the central notion of " amplitude" of the public debate.

\section{INDEX}

Mots-clés : amplitude, conflit de nomination, débat public, portée stratégique

Keywords : amplitude, conflict of naming, public debate, strategic effect 
AUTEUR

GILLES GAUTHIER

Université Laval (Québec) 